\title{
25 Das Öffentliche. Die Konzeptionen von John Dewey und Helmuth Plessner im Vergleich
}

An der Unterscheidung zwischen Privatem und Öffentlichem hängen gravierende Beweis- und Folgelasten in den modernen Gesellschaften, weshalb sie heiß umkämpft ist oder nivelliert wird. Man kann diese Unterscheidung überhaupt einebnen und auflösen, oder man kann sie verkehren, indem Privates als Öffentliches ausgegeben bzw. umgekehrt Öffentliches in Privates verwandelt wird, oder man kann diese Unterscheidung zwischen Privatem und Öffentlichem in einer Trennung verfestigen, sodass zeitlich keine neue Ausbildung dieser Unterscheidung erfolgen kann. Die Auflösungen, Verkehrungen und Verfestigungen der Unterscheidung zwischen Privatem und Öffentlichem sind die bekannten Themen von Hannah Arendt (Arendt 1981b), Richard Sennett (Sennett 1983) und Jürgen Habermas (Habermas 1981) in der Thematisierung des Politischen aus dem Öffentlichen im Unterschied zum Privaten.

Aber die Idee, dass das Politische nur vom Öffentlichen her auf angemessene Weise thematisiert werden kann, kommt auf völlig verschiedenen, einander nicht beeinflussenden Wegen durch John Dewey und Helmuth Plessner in den 20er Jahren des vorigen Jahrhunderts zustande. Beide liegen nicht nur geschichtlich früher als die heutige Diskussion, sondern bleiben für diese auch systematisch anregend (Krüger 2009a). Bevor man Fehlentwicklungen oder, mit Bruno Latour gesprochen, auch Neufassungen der Unterscheidung zwischen Privatem und Öffentlichem ausführen kann, braucht man logisch die Unterscheidung und den Zusammenhang der beiden unterschiedenen Seiten selbst. $\mathrm{Zu}$ Recht verweist Latour auf John Dewey, dessen Konzeption er dann aber zu schnell wieder aufgibt in seinem Vorschlag $\mathrm{zu}$ einem Parlament der Dinge (Latour 1999, 320, 350). Auch Habermas hatte bereits wie Latour, wenngleich auf andere Weise, eine neue Gewaltenteilung zugunsten des Öffentlichen gefordert, die über die klassische Gewaltenteilung zwischen Legislative, Exekutive, Juridikative und Öffentlichkeit innerhalb des politischen Handlungsbereichs moderner Gesellschaften deutlich hinausgeht. Sie bezog sich nicht nur auf den Primat der Demokratie über die Kapitalökonomie, sondern den Primat kultureller Pluralität über die Politik (Habermas 1985b).

Auch Dewey und Plessner setzen nicht erst in dem auf Politik spezialisierten Handlungsbereich mit ihrer Unterscheidung ein, sondern viel grundsätzlicher. Wie kann die Unterscheidung zwischen Privatem und Öffentlichem im Vergleich der Konzeptionen von Dewey und Plessner verstanden und begründet werden? Zur Beantwortung dieser Frage durchlaufe ich zunächst Deweys Öf- 
fentlichkeitskonzeption, sodann die von Plessner, um schließlich in einen Vergleich beider zu münden.

\subsection{Zu Deweys Öffentlichkeitskonzeption}

Auf den ersten Blick scheint bei John Dewey, in seinem Buch The Public and its problems (1927), die Öffentlichkeit nur ein Durchgangsstadium auf dem Weg von der Great Society zur Great Community zu sein (Dewey 1996). Die kapitalistische Industrie- und Konsumgesellschaft, eben Great Society, zeitigt direkte und indirekte Folgen. Soweit die Folgen von ihren Verursachern und den von diesen Folgen Betroffenen direkt wahrgenommen, beurteilt und geregelt werden können, handele es sich um Privates, so Dewey. Dies ist also ein sehr weiter Begriff des Privaten, der sich nicht auf Familiäres, Individuelles oder gar Intimes reduzieren lässt. Nach ihm können leicht Hunderte von Menschen privat miteinander interagieren, d. h. sich assoziieren und Transaktionen vollführen. Fallen aber die Verursacher der Folgen und die von den Folgen Betroffenen auseinander, und zwar für die Wahrnehmung, Beurteilung und Regulierung der Folgen, so handele es sich um indirekte Folgen. Werden nun solche indirekten Folgen für die von ihnen Betroffenen zu einem Problem, sowohl im guten Sinne, dass man die Folgen mehren möchte, als auch im schlechten Sinne, dass man diese Folgen mindern möchte, dann brauche man faktischer und legitimer Weise eine Öffentlichkeit dieser, von indirekten und problematischen Folgen Betroffenen. Unter dem Begriff der Öffentlichkeit firmiert dann alles, was zunächst einmal diese indirekten und problematischen Folgen wahrnehmbar, beurteilbar und regulierbar für die Betroffenen macht. Dadurch kann sich überhaupt ein Interesse der Betroffenen und darüber vermittelt der Verursacher herausbilden (zusammengefasst aus ebd., 20-22, 26-28, 31).

$\mathrm{Zu}$ dieser jeweils bestimmten Öffentlichkeit gehört aber auch die politische Organisation und Repräsentation dieser Interessen im Kampf mit anderen Interessen. Sowohl die Verursacher der indirekten Folgeprobleme für andere können ein Interesse daran haben, dass die Folgeprobleme privatisiert werden, als auch die Vertreter der jeweiligen Öffentlichkeit könnten als Privatpersonen Vorteile von der Privatisierung dieses öffentlichen Interesses haben. Insofern hängt die Herausbildung und Stabilisierung einer bestimmten Öffentlichkeit von vornherein von ihrer anspruchsvollen Demokratisierung nach innen und außen ab. Viele Öffentlichkeiten scheitern daran, dass sie von außen und innen gleichsam unterwandert werden. Für ihre Herausbildung und Stabilisierung ist es wichtig, dass sich die verschiedenen, je nach indirektem Folgeproblem gebildeten Öffentlichkeiten auch überlappen und gegenseitig verstärken, gleich- 
sam verdichten können. Solche Tendenzen können zu einer Great Community führen, welche die Great Society kanalisiert. Strukturell denkt Dewey diesen Prozess als einen offenen, der stets erneut anhand indirekter Folgeprobleme durch Öffentlichkeiten zu dieser Communitybildung hin durchlaufen werden kann (siehe ebd., 42-44, 75f, 95f, 112, 128f, 179-181).

Die Great Community ersetzt bei Dewey nicht die Great Society, aber reguliert deren indirekte Folgeprobleme. Erstere ist nicht als die geschichtsphilosophische Abschaffung oder auch nur Überwindung letzterer zu verstehen. Beide verhalten sich funktional wie Frage und Antwort zueinander. Die Öffentlichkeiten haben die Aufgabe, aus der Vielzahl möglicher Fragen und Antworten in ihrer Überlappung die politisch relevanten zu verdichten und herauszuheben. Sie führen diejenigen Alltags- und Expertenkulturen zusammen, die für die jeweilige Lösung des indirekten Folgeproblems einschlägig sind. Dadurch ermöglichen Öffentlichkeiten eine experimentelle Zuordnung zwischen Frage und Antwort im politischen Interessenkampf.

Diese grobe Skizze legt den Akzent darauf, dass die Unterscheidung zwischen Privatem und Öffentlichem in den funktionalen Zusammenhang zwischen Great Society und Great Community gleichsam eingelagert ist. Dewey glaubt zwar, dass er eine derart „funktionale“ Hypothese auch auf andere Kontexte übertragen kann, aber er will und kann diese modifizierende Übertragung nicht in seinem Buch leisten (ebd., 46f., 61, 71). Er gehört zweifellos zu den Begründern einer reflexiven Modernisierung (Ulrich Beck), wenn damit gemeint ist, dass eine klassisch moderne, also in viele autonome Handlungsbereiche zerfallende Gesellschaft Folgen hat, direkte und indirekte, die durch die Unterscheidung privat-öffentlich bewältigt werden müssen. Dieses „Müssen“ bei Dewey ist kein Automatismus oder Determinismus, aber weit mehr als ein bloßes Sollen, da er die Alternative klar vermerkt: Wenn es nicht zu, wie Dewey 1946 in seinem neuen Vorwort wiederholt, Weltwirtschaftskrisen und Weltkriegen kommen soll. Diese scheinbaren Naturkatastrophen stellten hausgemachte Folgen einer problematischen Modernisierung dar (ebd., 112-118, 170-173, 183, 197f.).

Was Dewey also neue öffentliche Lernprozesse nennt, dient der Lösung der indirekten Folgeprobleme der klassisch autonomen Modernisierung. Die vielen Autonomien mögen nicht vormodern abgeschafft werden, sondern anhand der indirekten Modernefolgen durch Interpenetrationen, also durch wechselseitige Durchdringungen der Handlungsbereiche und Handlungsarten, korrigiert werden. Deweys zweite Moderne steht nicht im Zeichen der traditionell modernen Autonomien, sondern innovativer Interpenetrationen, die die Folgeprobleme der ersten Modernisierung lösen. Dafür ist die neue Unterscheidung zwischen privat 
und öffentlich kardinal. Sie kann sich nicht mehr an die traditionell vorgegebenen Autonomien - vom Individuum über die Wirtschaft und Wissenschaft bis zur Kunst und Politik, etwa dem Nationalstaat - halten, und zwar der Folgen wegen, vor allem wegen der indirekten Folgeprobleme, die, werden sie nicht gelöst, die Wucht von Naturkatastrophen annehmen, wenn sie nicht gelöst werden.

Die ganze Unterscheidung privat-öffentlich kritisiert die Autonomie der Politik im Sinne einer eigenen Handlungssphäre neben anderen derart autonomen Handlungssphären zugunsten der experimentellen Neuausbildung des Politischen aus dem Öffentlichen. Dass Politik und Staat ein eigenes „Wesen“ haben sollen, ist für Dewey ein „Mythos“, und dass dem Nationalstaat „Souveränität“ zukomme, „ist lediglich eine vollständige Leugnung politischer Verantwortung“ (ebd., 24f, 183). Geschichtliche Prozesse verlaufen nicht nach der „Theorie der kausalen Urheberschaft“ oder gar „Autorschaft“ individueller oder kollektiver „Willen“, wonach man Autonomie gedacht habe (ebd., 30f., 58, 139). Die „Schüler von Lenin und Mussolini wetteifern mit den Kapitänen der kapitalistischen Wirtschaft in dem Bestreben, eine Formung von Dispositionen und Ideen vorzunehmen, welche zu einem vorgefassten Ziel führen wird“ (ebd., 167). Aber diese Kontrollmythen abstrahierten von der Geschichtlichkeit der Menschennatur in deren Generationenfolge: „Denn das vermehrte Wissen über die menschliche Natur würde die Beschaffenheit der menschlichen Natur direkt und auf unvorhersehbare Weise verändern und zur Notwendigkeit neuer Regulierungsmethoden führen, und so fort in alle Ewigkeit“ (ebd., 165).

Logisch ergibt sich aus der Transformation bestimmter Autonomien durch bestimmte Interpenetrationen, erfolgt sie nur oft genug von neuem, dass früher oder später Interpenetrationen auf Interpenetrationen rekurrieren, um anhand von indirekten Folgeproblemen zu Lösungen zu gelangen. So ist in der Tat Deweys Logik von Anfang an angelegt, dass nämlich inquiries auf inquiries folgen, so dass man sich heute immer schon an dem „principle of the continuum of inquiry“ (Dewey 1991, 3) orientieren möge. Die deutsche Übersetzung als „Theorie der Forschung“ (Dewey 2002) ist unglücklich, weil sie Dewey in die Nähe von Karl Raimund Popper bringt, der in seiner Logik der Forschung (1935) die Autonomie der Wissenschaft durch ihre Demarkation von nichtwissenschaftlichen Handlungssphären sicherstellen wollte. Demgegenüber kommen wir laut Dewey nicht mehr aus den Untersuchungsprozessen heraus, die bei ihm keineswegs mit der klassisch-modern etablierten Autonomie der Erfahrungswissenschaft verwechselt werden dürfen. Inquiries stellen Interpenetrationen dar, nämlich der juristisch öffentlichen Prozedur der Urteilsfindung (im Anschluss an den common sense mit biologischer und existentieller Matrix) mit 
erfahrungswissenschaftlichen und technologisch-operativen Expertisen (siehe Krüger 2000d).

In dieser Art und Weise der Interpenetration, die inquiry heißt, steht die intelligente Rekonstruktion als Mittel, eben die Instrumentierung, im Vordergrund. Aber Dewey wehrt sich gerade gegen die Trennung von Mittel und Zweck, von Instrumentierung und Wertbindung. Gesellschaftliche Kommunikation gebe es in dem Maße, in dem Instrumentierung und Finalisierung öffentlich geteilt werden können:

Kommunikation ist auf einzigartige Weise sowohl Mittel wie Ziel. Sie ist Mittel, insofern sie uns von dem andernfalls überwältigenden Druck der Ereignisse befreit und uns in den Stand setzt, in einer Welt von Dingen zu leben, die Sinn haben. Sie ist Ziel als Teilhabe an den Objekten und Künsten, die für eine Gemeinschaft von Wert sind, eine Teilhabe, durch die Bedeutungen im Sinne der Kommunion erweitert, vertieft und gefestigt werden. (Dewey 1995, 201)

Die andere Art und Weise von Interpenetration, welche die Zweckbildung und Wertebindung durch Werterfüllung betrifft, nennt er die im weiten qualitativen Sinne ästhetische, darunter künstlerische und religiöse Erfahrung (Dewey 1980). Auch hier wird öffentlich am Material verfahren, intelligent rekonstruiert, aber so, dass eine gemeinsame ästhetische Erfahrung entsteht, die durch signifikante Symbole herausgehoben wird. Sie dient einer Bindung an Werte, die, wie Hans Joas herausgearbeitet hat, das jeweilige Selbst überschreiten, ja, überhaupt die dem Menschen mögliche Selbstbildung transzendieren können (Joas 1997, 7. Kap.).

Wir haben es also in Deweys Spätwerk mit zwei Interpenetrationsrichtungen zu tun, in die Richtung der Instrumentierungen nach gelebten Werten und in die Richtung der consummations, $d$. h. der im Vollzug erlebten Vollendung ästhetischer Erfahrung, vor allem dann, wenn letztere für die Gemeinschaftsbildung signifikant wird. Durch die Auszeichnung dieser beiden Interpenetrationsrichtungen, die es gelte, in der gesellschaftlichen Kommunikation zu integrieren, ist klar, dass Dewey mit Interpenetration nicht gemeint hat, dass sich die kapitalistische Wirtschaft die anderen autonomen Prozesse der Wissenschaft, Politik und Kunst einverleibt. Diese Art von Kritik, z. B. die neoliberale Kritik an modernen Autonomien im Namen der Interpenetration kann sich nicht auf ihn berufen. Der philosophische Maßstab bleibt bei Dewey die gesellschaftliche Kommunikation, soweit sie die Instrumentierungen und Finalisierungen nach letztlich ästhetischen Vollendungen der Selbsttranszendierung integriert. Daher Deweys Kritik an dem klassisch modernen Selbstverständnis, dass Menschen eben nur „kleine Götter“, d. h. allein keine großen, wohl aber doch Götter seien. Deweys Wertekritik am Ende von Experience and Nature kulminiert in seiner 
Kritik am kleinen Gott: Ob klein oder groß, der Mensch könne nicht die Rolle Gottes übernehmen (Dewey 1995, 405). Die klassisch autonome Modernisierung ist eine Fehlmodernisierung, weil sie ihre stets erneuten Grenzen, eben ihre indirekten Folgeprobleme, nicht aus sich meistern kann. Dies ist heute ökologisch offenbar.

So wenig man Dewey einen Neoliberalen nennen kann, so wenig kann man ihn als einen Neokommunitaristen bezeichnen. Was er die Great Community nennt, geht ja aus der gesellschaftlichen Kommunikation von verschieden bestimmten Öffentlichkeiten hervor, die ihrerseits auf indirekte Folgeprobleme der Great Society antworten. Es handelt sich also keinesfalls um eine Herkunftsoder Traditionsgemeinschaft, sondern um eine zunächst potentielle Gemeinschaft all derjenigen, die positiv und negativ von bestimmten indirekten Folgeproblemen betroffen sind. Der Startpunkt in dem Modell ist die Aufgabe einer neuen Gemeinschaftsbildung unter Leuten, die bislang weder wussten noch bewusst erfahren haben, dass sie zu Betroffenen dieser Art von Folgen gehören. Die Gemeinschaftsbildung rekrutiert sich funktional aus Alltags- und Expertenkulturen, und die Funktion der Öffentlichkeiten reicht bis in das Experimentalverständnis der Politik hinein. Eine Ausnahme bilden nur die lokalen Gemeinschaften, die traditional sein können, es nicht müssen, und die letztlich als anthropologisch nötige Gemeinschaftsformen der Nachbarschaft verstanden werden. Ohne dass Menschen als Lebewesen sich räumlich und zeitlich begegnen können, für einander zu Beobachtern und Teilnehmern leibhaftig werden können, gäbe es keine Generationenfolge. Kein Zweifel, am Ende steckt hinter allen Kategorien, die Dewey in seiner Öffentlichkeitskonzeption verwendet, eine philosophische Anthropologie (Krüger 2001). Sie fundiert die reflexive Moderne durch Interpenetrationen, was hier nicht mehr Thema ist: „Das Gesicht ist ein Zuschauer, das Gehör ist ein Teilnehmer“ (Dewey 1996, 181; zur Aktualität von Deweys Öffentlichkeitskonzeption insgesamt Antic 2018).

\subsection{Zu Plessners Öffentlichkeitskonzeption}

Plessner entwirft 1924 seine Öffentlichkeitskonzeption in dem Buch Grenzen der Gemeinschaft. Zur Kritik des sozialen Radikalismus. Unter „Radikalismus“ versteht er exklusive Entweder-Oder-Alternativen, die nichts Drittes gestatten. Die Seiten einer Alternative werden dann radikal verstanden, wenn sie de facto und de jure nur als sich gegenseitig ausschließend aufgefasst werden, ohne dass diese Seiten in einer anderen Hinsicht auch als einander bedürftiger Zusammenhang behandelt werden. Im vorliegenden Falle geht es um den Dualismus Gemeinschaft oder Gesellschaft, die radikal so gehandhabt wird, dass es grund- 
sätzlich nur entweder Gemeinschaft oder Gesellschaft geben dürfe (Plessner 1981b, 14f., 17f.). Dieser Fehlalternative stellt Plessner als Drittes eine Individualität (ebd., 32, 42, 44, 60-64) gegenüber, die sowohl der Vergemeinschaftung als auch der Vergesellschaftung bedarf: „In uns selbst liegen neben den gemeinschaftsverlangenden und gemeinschaftsstützenden die gesellschaftsverlangenden, distanzierenden Mächte des Leibes nicht weniger wie der Seele, in jeder Sozialbeziehung wartet die eine, wenn noch die andere gilt, auf ihre Erweckung“ (ebd., 115).

Die Individualität wird quasi aristotelisch als eine leibliche anima verstanden, welche einer Zweideutigkeit zwischen Ontischem und Ontologischem unterliege. Sie bedürfe zwar in ihrem Dasein (ontisch) schon immer eines Verständnisses von Seiendem (ontologisch) und umgekehrt auch in ihrem Verstehen von Seiendem (ontologisch) eines Soseins (ontisch). Aber die Zuordnung zwischen den Bestimmungen von Seiendem (ontisch), zum Beispiel einer bestimmten Haar- und Körperfarbe, und dem Verstehen von Seiendem (ontologisch), zum Beispiel als Zeichen der Schichtzugehörigkeit und des Bildungsgrades, sei eben nicht eindeutig, sondern zweideutig oder gar mehrdeutig. Plessner spricht kurz von der „ontisch-ontologischen Zweideutigkeit“ leiblicher Seelen (ebd., 55f, 61f., 64, 66, 92). Eine solche Person könne weder physisch noch psychisch auf eine endgültige Weise positiv festgelegt werden, da die ihr wesentliche Lebendigkeit nur in drei Haltungswechseln im Verhalten gedeihen könne. Sie müsse kognitiv zwischen der Orientierung an Realität und der an Irrealität, ästhetisch zwischen Naivität und Reflexion und ethisch zwischen Geltungsdrang und Verhaltenheit wechseln können (ebd., 66-75, 105f.). Gäbe es diese Verhaltensambivalenzen nicht, bestünde auch nicht das uns bekannte Problem, wie in dem räumlichen und zeitlichen Verhaltensfluss situativer Einschränkungen auf eine bestimmte Aktualität die Würde der Person, also das Ganze ihrer physischen, psychischen und geistigen Potentiale, bewahrt werden kann. „Würde betrifft stets das Ganze der Person, den Einklang ihres Inneren und Äußeren, und bezeichnet jene ideale Verfassung, nach der die Menschen streben, die aber nur wenigen verliehen ist“ (ebd., 75f.). Die Konkordanz, die „Einstimmigkeit zwischen Geist und Leben ist nicht ein Freibrief der Raserei, sondern die Bürgschaft der menschlichen Würde, die nur durch Maßlosigkeit zerstört werden kann. Maß und Begrenzung ist das Höchste für menschliches Streben“ (ebd., 131).

Für das Ausbalancieren der einander entgegengesetzten Verhaltensrichtungen brauche Individualität soziokulturelle Rollen, ein Maskenspiel. Dank einer Rolle kann sie sich verdoppeln, sowohl vor anderen, d. h. in öffentlicher Richtung, als auch vor sich selbst, d. h. in privater Richtung. Nicht die Haut des 
Organismus bildet die Grenze des Menschen, sondern der Verhaltensraum und die Verhaltenszeit der Rolle, die außerhalb des Organismus in der Mitwelt liegt. Eine persona bewegt sich als soziokultureller Körper zwischen den leiblichen Seelen und ihren physischen Körpern im Hinblick auf andere Personen. Von den soziokulturellen Rollenkörpern her gesehen entstehen zwei Verhaltungsrichtungen. Durch das Medium dieser habituellen Masken kann man sich zu den anderen hin und von ihnen her zu sich zurück verhalten. Und von mir her betrachtet kann ich mich in die Rolle hinaus und aus ihr zu mir zurück bewegen. Das Selbst der personae könne nur in dieser Doppelstruktur, gleichsam vor und hinter der Maske, und in beiden Richtungen, in und mit der Rolle hinaus und aus diesem Außen zurück in je mein Inneres, entstehen und gedeihen (ausführlich siehe Krüger 1999, im vorliegenden Band Zweiter Teil).

Schaut man von dieser personalen Doppelstruktur mit ihren beiden Verhaltensrichtungen auf die Unterscheidung zwischen Gemeinschaft und Gesellschaft, fällt sie anders als im sozialen Radikalismus aus. Wer der Individualisierung unterliege, könne diese Erfahrung seiner Unteilbarkeit und Einzigartigkeit nur machen, indem er bzw. sie diese Doppelstruktur in beiden Richtungen durchläuft, so Plessner. Die ontisch-ontologische Zweideutigkeit verlangt die Entfaltung eines Verhaltensspektrums in Raum und Zeit, um die genannten Ambivalenzen ausleben und aus diesem Ausdruck auf sich zurückkommen zu können. In ihr sind sowohl Gemeinschaftsverlangen als auch Gesellschaftsverlangen fundiert, und zwar in rhythmischen Wechseln der Lebensgeschichten als auch in der Folge der Generationen. Sich leiblich und seelisch individualisierende Personen brauchen familiare Gemeinschaftsformen, in denen nach Gefühlswerten primär unmittelbar interagiert werden kann, als auch geistige Gemeinschaftsformen, in denen - durch Bildung und Dritte vermittelt - nach Sachwerten interagiert werden kann. Beide Gemeinschaftsformen sind nötig, damit die Verhaltensambivalenzen überhaupt Konturen in habituellen Haltungen zum Leben gewinnen können (Plessner 1981b., 45-52). Hat man diese historisch bestimmten Konturen, fragt sich aber weiter, was an ihnen gemessen im Alltäglichen und Außergewöhnlichen als unbekannt, als unverbunden, als andersartig, ja, als fremd gelten muss. Da man für diese phänomenalen Begegnungen weder über familiäre noch über sachliche Gemeinschaftswerte verfügt, geht es nun darum, eine solchen Werten äquivalente Verhaltensbildung zu ermöglichen. Plessner spricht von der Verhaltensermöglichung in Werteferne, also in der Ferne von den jeweils vertrauten Gemeinschaftswerten, und in Wertäquivalenz, also in einer Äquivalenz für jene Gemeinschaftswerte, im Verhalten der unter einander Unbekannten und Unverbundenen, füreinander Anderen und Fremden (siehe ebd., 79-81, 95f., 133). 
Das Überraschende an der Begriffswelt der Schrift von 1924 besteht darin, dass er alle Interaktionsmöglichkeiten unter Bedingungen der Werteäquivalenz sowohl mit Gesellschaft als auch mit Öffentlichkeit identifiziert (ebd., 95-112, 115-117). Diese Identifikation leuchtet nur historisch, nicht systematisch ein. Historisch verteidigt er nicht die Gesellschaft im spezifisch kapitalistischen Sinne. Es geht ihm nicht wie Dewey um die Great Society. Plessner verteidigt historisch die abendländisch-westliche Zivilisation gegen die deutsche Kulturgemeinschaft und gegen die allein klassenbedingte Weltanschauungsgemeinschaft (ebd., 31, 35, 38, 40f., 42f., 93f.). Die Doppelstruktur der personae ist Zivilisationsmaß, auch gegen den bourgeois, und kann daher im Text mit aristotelischen, höfischen, neuzeitlichen oder modernen Beispielen erläutert werden. Man denke an seine Unterscheidung zwischen Zeremoniell und Prestige oder zwischen Diplomatie und Takt. Was systematisch durchläuft, ist die personale Doppelstruktur in beiden Bewegungsrichtungen. Plessner entfaltet sie im Spätwerk in klarer Unterscheidung von den Funktionswerten moderner Gesellschaften, so in der Frage nach der Conditio humana (Plessner 1983e, 198-203) und über die Anthropologie des Schauspielers (Plessner 1982i, 406, 416).

Worin besteht also der systematisch bedeutsame Kern von Plessners Öffentlichkeitskonzeption? In der Anerkennung von Pluralität im Gefolge der Individualisierung der Personen, d. h. deren Doppelstruktur zwischen Privatem und Öffentlichem. Selbst wenn es eine einzige Ursprungsgemeinschaft von Personalität gegeben haben sollte oder künftig geben würde, kann sich Personalität nicht anders als durch ihre leibliche und seelische Individualisierung der personalen Doppelstruktur im Leben halten. Eine Person variiert im leiblichen Vollzug die Struktur ihrer Verdoppelung in Öffentliches und Privates. Solange sie lebt, ist sie in ihrem Verhalten nicht endgültig festgestellt und bedarf daher der Bewahrung ihrer Würde. In gewissem Sinne gilt dies auch für ihre Erinnerung durch andere. Zumindest in der Folge der Generationen würden andere als die ursprünglichen Möglichkeiten zur Gemeinschafts- und Gesellschaftsbildung zum Tragen kommen können. Öffentlichkeit ermöglicht Verhalten unter einander Unbekannten, die sich unbekannt bleiben können, unter einander Unverbundenen, die nicht verbunden werden müssen, unter einander Anderen, die nicht die gleichen Werte teilen müssen, unter einander Fremden, die sich fremd bleiben können. Sie zivilisiert den Kampf auf Leben und Tod durch ein Maß an gegenseitiger Schonung. Die Zukunft bleibt eine plurale Herausforderung am Rande der Gewalt, weil selbst die Pluralismusdoktrin vom neutralen Staat auf sie nicht abschließend antworten kann.

Plessners späteres Buch Macht und menschliche Natur (1931) handelt expressis verbis von der „wertedemokratischen Gleichstellung aller Kulturen“ 
(Plessner 1981d, 186) im Namen einer künftigen Zivilisiertheit, die den anderen und fremden Menschen nicht am allein eigenen Wertemaßstab misst. Die von Plessner (mit Wilhelm Dilthey und Georg Misch) geteilte Lebensphilosophie bezeugt „ihr Europäertum, das im Zurücktreten von seiner Monopolisierung der Menschlichkeit das Fremde zu seiner Selbstbestimmung nach eigener Willkür entbindet und mit ihm in einer neu errungenen Sphäre von Freiheit das fair play beginnt“ (ebd., 228). Die Individualisierung der Personalität bringt leiblich nicht nur die etablierten Standards zum Ausdruck, sondern variiert diese und weicht von ihnen ab. Dies ist keine Frage der allein bewussten Entscheidbarkeit. Nur wer bewusstseinsphilosophisch denke, so Plessner, nehme an, dass in der Geschichte selbstbewusst über die Geschichte entschieden werden könne. Gelänge letzteres, wäre das Ende der Geschichte erreicht worden, wären wir vor Ereignissen endgültig sicher, hätte sich das Problem der Zurechenbarkeit im Sinne der „säkularisierten Vergottung des Menschen“ (ebd., 150) aufgelöst. Bewusstsein ist aber nur eine Verhaltensdimension, die sich nicht sich selbst unterstellen kann, ohne in Verhaltensparalyse zu geraten. Plessner wehrt die Übertragung des Autonomiemodells vom Selbstbewusstsein auf das Ganze der Geschichte ab. Er geht mit dem Streit über die Frage, wem das Primat über den Geschichtsprozess im Ganzen gebühre, anders als aus der Sicht von überverallgemeinerten Teilautonomien um. Weder der Philosophie noch der Anthropologie noch der Politik als Teildimensionen komme das Primat über den künftigen Geschichtsprozess im Ganzen zu. Für ihn gelte, sowohl faktisch als auch normativ, das Prinzip der „Nichtentscheidbarkeit des Vorrangs“ (ebd., 218).

Personale Lebewesen sind solche, die gemessen an ihrer Bestimmtheit durch Rollen in eine Relation zu ihrer künftigen Unbestimmtheit geraten, solange sie leben. Dies bedeutet, dass das personale Selbst seinem Eigenen noch unbekannt, unverbunden, anders und fremd werden kann. Das Selbst der personae ist nicht das Eigene, sondern die oben genannte Doppelstruktur in beiden leiblichen Bewegungsrichtungen, in deren Dynamik Änderungen möglich bleiben. Jetzt versteht man auch, warum Plessner 1924 in den Grenzen der Gemeinschaft sagt, einer der größten Fehler bestünde darin zu denken, die Gesellschaft laufe außen um die Gemeinschaft herum, statt mitten „durch sie hindurch“ (Plessner 1981b, 115). Gesellschaft bricht in der Individualisierung der personalen Doppelstruktur in beiden Bewegungsrichtungen hervor. Und dies sei keine Frage des guten oder schlechten Willens. Ähnlich heißt es am Ende von Plessners Naturphilosophie in den Stufen des Organischen und der Mensch (1928), Personalität werde in einer Mitwelt ermöglicht, welche strukturell gesehen nicht zwischen Eigenem und Anderem zu wählen habe, nicht einmal zwischen Singular und Plural. Solche Differenzen zwischen Eigenem und Anderem 
oder Singular und Plural stellten schon, im Resultat historischer Kämpfe, Auslegungen dafür dar, wie die Mitwelt geschichtlich eingeschränkt werde (Plessner 1975, 301). Diesen Einschränkungen sei indessen ein Strukturpotential vorausgesetzt, das sich durch den Bruch zwischen physischen, psychischen und geistigen Verhaltensdimensionen auszeichne. Dieser Hiatus müsse zwar immer erneut hier und jetzt verschränkt werden, um leben zu können, er könne aber nicht endgültig überwunden werden, weil man dafür außerhalb des Lebens über ihm zu stehen kommen müsste.

In Plessners Philosophischer Anthropologie wird die personale Lebensführung hier und heute aus der künftigen Geschichtlichkeit ermöglicht. Statt des Endes der Geschichte in diesem Gemeinschaftsideal oder in jenem Gesellschaftsideal wird diese Ermöglichung zur stets erneuten geschichtlichen Aufgabe, den naturphilosophisch freigelegten Bruch zu verschränken. In dieser geschichtlichen Verschränkungsdynamik eines exzentrischen Bruches mit der leiblichen Konzentrik zwischen Organismus und Umwelt besteht die anthropologische Konstante. Plessner nennt sie die Unergründlichkeit des Menschen, kurz: den homo absconditus. Die Mitwelt ermögliche eine „Wirform des eigenen Ichs“, welche dem Verhalten zwischen ihm, dem Anderen, und mir entspreche: „Zwischen mir und mir, mir und ihm liegt die Sphäre des Geistes“ (Plessner 1975, 303). Sie ist an den Modus ihrer öffentlichen Realisierung gebunden (ebd., 345).

In der Dynamik des Durchlebens der personalen Doppelstruktur wird einem Anderes eigen und Eigenes anders. Sie wird in den Autonomieforderungen künstlich auf die Feststellung des je Eigenen fixiert. Plessners Kritik an modernen Autonomisierungen besteht darin, dass in ihnen die je eigene Willensbildung über deren Kollektivierung einer Logik der Selbstvergottung des Menschen folgt. Dadurch werde die je individuelle Würde einer Person der jeweiligen Mehrzahl von Organismen geopfert, die man für dieses Gemeinschaftsideal oder jenes Gesellschaftsideal lebenspolitisch instrumentiert. Man missverstehe die Struktur der Personalität, das höchste Gut der Zivilisation, wenn man es utilitaristisch von der Anzahl der Organismen abhängig machen wollte (Plessner 1975, 315). Die personale Verschränkung des Verhaltensbruchs geschieht gegenüber Anderem nicht grundsätzlich anders als gegenüber Eigenem, weil die Person ja gerade in keinem Organismus steckt. Sie ist diejenige Position, die aus der Interaktion eines Organismus mit seiner Umwelt herausgesetzt ist. Sie geht also über das tierliche Niveau hinaus, in dem sich ein zentrischer Organismus (Organismus mit Gehirn) und eine an Bewusstsein gebundene Umwelt aufeinander einspielen. Dieses tierliche Verhaltensniveau nennt Plessner eine zentrische Positionalitätsform. Erst die exzentrische Positionalitäts- 
form ermögliche aber menschliches Leben. In ihr kann zugunsten von - geistig in einer Mitwelt geteilter - Personalität exzentriert werden. Von dieser Exzentrierung her müsse dann jedoch die zentrische Positionalitätsform neu, d.h. soziokulturell eingerichtet werden, weil die zentrische Organismusform einer zentrischen Verhaltensform bedarf. Gegen diese künstliche Einschränkung der Zugehörigkeit zum Kreis der Personen durch eine bestimmte soziokulturelle Empirie im Gegensatz zu anderen solcher Empirien legt Plessner dasjenige Potential frei, das dem sozial- und kulturgeschichtlichen Wandel in der Interpretation der personalen Welt vorausgesetzt ist: „Real ist die Mitwelt, wenn auch nur eine Person existiert, weil sie die mit der exzentrischen Positionsform gewährleistete Sphäre darstellt, die jeder Aussonderung in der ersten, zweiten, dritten Person Singularis und Pluralis zu Grunde liegt“ (Plessner 1975, 304).

Der einzige, für das Individuum aber existentielle Unterschied zwischen der Struktur des Verhaltens von Anderen zu mir und von mir zu mir besteht darin, dass es nur an den einen, eben seinen Organismus gebunden bleibt. Denn die Exzentrierung der Positionalität geht nicht ohne die künstliche Fortsetzung einer zentrischen Positionalität. Diese Bindung an den eigenen Körper stellt eine zivilisationsgeschichtliche Grenze für alle Verschmelzungsphantasien mit Anderen dar. Diese Formulierung ist indessen eine Erkenntnis in Kategorien des Körperhabens, nicht des Leibseins. In der personalen Doppelstruktur gibt es grundsätzlich zugleich die Möglichkeit, sich mit andren Körpern leiblich identifizieren zu können. Anderenfalls wäre sozial geteilte Kultur überhaupt nicht möglich.

\subsection{Vergleich der beiden Öffentlichkeitskonzeptionen}

Was Plessner im Kern Öffentlichkeit nennt, die Ermöglichung des Verhaltens unter einander Unbekannten, Unverbundenen, Anderen und Fremden, taucht bei Dewey unter dem Begriff der indirekten Folgeprobleme auf, deren Bewältigung Öffentlichkeit braucht. Diese Verständnisse des Öffentlichen im Unterschied zum Privaten sind logisch vergleichbar. Die indirekten Folgeprobleme sind unbekannt, unverbunden, anders und fremd gemessen an den bisher habitualisierten Erwartungen, die man gemäß der klassisch modernen Autonomie in Handlungsbereichen hegt. Auch was Plessner die einander Unbekannten, Unverbundenen, Anderen und Fremden nennt, ist umgekehrt ein indirektes Folgeproblem der positiven Fassung von bestimmten Gemeinschafts- und Gesellschaftsformen. In beiden Konzeptionen wird der Positivismus der klassisch modernen Autonomien, d. h. die positive Selbstbestimmung und Selbstverwirklichung von individuellen, kollektiven oder funktionalen Akteuren, deutlich 
begrenzt. Er wird in Frage gestellt durch die Einführung der Relation zur eigenen Unbestimmtheit aus der Zukunft heraus. Was in der Vergangenheit als eine positive Leistung durch individuelle, kollektive und funktionale Autonomisierungen von Teilprozessen erschien, ist nur ein Moment und eine Tendenz in einem geschichtlichen Prozess, der im Ganzen so offen ist, dass er künftig auch schief gehen kann, werden nämlich einerseits nicht die indirekten Folgeprobleme bearbeitet und wird nicht andererseits dafür eine strukturelle Vorkehrung getroffen, die Würde der lebenden Personen schon heute in Zukunft wahren zu können. In beiden Konzeptionen werden die klassisch modernen Autonomievorstellungen dafür kritisiert, dass sie einer Logik der „Selbstvergottung“ des Menschen (Plessner) bzw. der Logik vom „kleinen Gott“ (Dewey) folgen, welche Menschen in der Übernahme der Rolle Gottes überfordert. Beide Konzeptionen resituieren die Unterscheidung zwischen Privatem und Öffentlichem in einem geschichtlichen Prozess, den kein Subjekt von Selbstbewusstsein, ob individuell, kollektiv oder funktional, als Ganzen in der Hand haben kann. Daher münden beide Konzeptionen konsequenter Weise in eine Philosophische Anthropologie, welche die anmaßende Selbstermächtigung zu quasi göttlichem Tun aufdeckt (continuum of inquiry, homo absconditus). Die anthropologische Konstante besteht nicht im Ende der Geschichte, sondern in der strukturellen Ermöglichung künftiger Geschichtlichkeit. ${ }^{1}$

Gleichwohl bleiben der Hauptfokus und die Begründungswege beider Konzeptionen verschieden. Sie verliefen ohnehin unabhängig voneinander. Für Dewey stehen die indirekten Folgeprobleme der Great Society im Vordergrund, die nicht ohne ihre öffentliche Kanalisierung durch eine Great Community überleben kann, sondern in Weltkrisen und Weltkriege abstürzt. Dies ist eine erneut überzeugende Aufgabe, auf die Plessner in seinem Buch Die verspätete Nation unter den Titeln des Hochkapitalismus und moderner Lebensmächte (Plessner 1982a; siehe im vorliegenden Band 17. Kapitel) eingeht, aber weniger elaboriert als Dewey. Plessner stellt sich einer anderen Aufgabe, nämlich die Zivilisationsleistung zu rekonstruieren, die es, wenngleich auf die Oberschichten begrenzt, seit den Achsenkulturen der Personalität gibt, d.h. einem geistig-kulturellen Potential seit der Achsenzeit für spätere Modernisierungen (auch im außerwestlichen Sinne von Karl Jaspers und Shmuel Eisenstadt). Diese Zivilisationsaufgabe liegt quer zu der Unterscheidung zwischen Vormoderne, Moderne und Postmoderne. Wenn die Moderne den Zivilisationsprozess abbricht, bricht alles, was

1 Die Philosophische Anthropologie von John Dewey wird deutlicher, wenn man nicht nur das Werk von G. H. Mead einbezieht, dass Dewey stets voraussetzt, sondern auch den Streit zwischen W. James und Ch. S. Peirce. Siehe Krüger 2001, 2. Teil. 
den Umgang mit Pluralisierung und Individualisierung der Personalität ermöglicht hat, auch zusammen. Mit ihrer Meisterung steht und fällt Zivilisiertheit, deren Gegenbegriff die Barbarei bildet, welche wir aus den Welt- und Bürgerkriegen des 20. Jahrhunderts kennen (siehe Joas 2005). 
